\title{
THE STRUCTURAL ANALYSIS OF MEDICINAL PLANTS AND THEIR USE TO TREAT THE GASTROINTESTINAL TRACT DISEASES
}

\author{
LiUBOV MAKHOVSKA, ViCTORIA GNIEZDILOVA, OKSANA NESPLIAK, VIRA BUNIAK
}

\begin{abstract}
Medicinal plants have long been used in the treatment of various diseases and diseases of the digestive system, in particular. They contain almost all biologically active substances, which gives them an advantage over synthetic drugs, and the possibility of long-term use. On the basis of processing of literature sources the systematical and biomorphological analyzes of herbs were carried out. We analyzed the medicinal plants which are used to treat diseases of the gastrointestinal tract, including four groups: stomach (atony, achillia, ulcer, gastritis), intestine (enteritis, colitis), liver and gallbladder (hepatitis A, cholangitis, cholecystitis, nausea) and dyspeptic disorders (vomiting, constipation, diarrhea, bloating). Each of these groups of illnesses is characterized by a specific list of medicinal plants with an indication of their life form. 110 species of medicinal plants were identified, which belong to 102 genera, 46 families, 33 orders, 4 classes and 3 divisions. Among studied herbs, the most numerous are herbaceous polycarpics - 60 species $(54.55 \%)$, while annuals have 21 species $(19.01 \%)$, trees - 9 species $(8.18 \%)$, shrubs - 8 species (7.27\%), biennials - 7 species (6.36\%), semi-shrubs - 4 species $(3.64 \%)$ and lianas - 1 species $(0.91 \%)$. The taxonomic and biomorphological structure of the studied plants is presented in general and analyzed in detail in each of the selected groups of diseases. Examples of plants that used in the treatment of various diseases of the gastrointestinal tract are given. It was established that for intestinal disease 28 species are used, liver and gallbladder - 47 species, stomach - 60 species and dyspeptic disorders - 75 species of herbs.

The article shows the results of taxonomic and biomorphologic analysis of medicinal plants that are used to treat the gastrointestinal tract diseases are based on literary data.
\end{abstract}

Keywords: medicinal plants, taxonomic structure, biomorphologic structure, gastrointestinal tract.

\section{INTRODUCTION}

Diseases of the gastrointestinal tract are common among the population and rank second only to cardiovascular disease. Almost every patient has some extent severe digestive disorders that require correction, although at the moment they may not manifest [3].

Herbs are especially useful in the treatment of gastrointestinal tract diseases. Almost the entire spectrum of pharmacological action is used, as there is a therapeutic effect on the condition of other 
organs and systems (nervous, immune, endocrine, etc.). Phytotherapeutic agents, even in a minimal amount, can significantly improve the condition of patients [6, 12].

The aim of our work was to conduct systematic and biomorphological analyzes of medicinal plants used to treat digestive system diseases.

\section{MAterials AND Methods}

The species composition of medicinal plants used in the treatment of digestive organs is given by "Opredelitel vysshyh rastenij Ukrainy" [8], "Atlasom likarskyh roslyn" [7]. Systematical structure is presented by A.L. Takhtajan [11], biomorphological - by I.G. Serebryakov [9], pharmacological characteristics - based on the analysis of literature sources $[1,2,4,5,6,12]$.

\section{RESULTS AND DISCUSSION}

To treat the digestive tract diseases 110 species of medicinal plants are used, which in taxonomic terms belong to 102 genera, 46 families, 33 orders, 4 classes (Equisetopsida, Pinopsida, Magnoliopsida, Liliopsida) and 3 divisions (Equisetophyta, Pinophyta та Magnoliophyta) (Tab. 1).

\begin{tabular}{|c|c|c|c|c|c|c|c|}
\hline \multirow{2}{*}{$\begin{array}{c}\text { Taxas } \\
\text { (division/class) }\end{array}$} & \multicolumn{2}{|c|}{ Families } & \multicolumn{2}{|c|}{ Genera } & \multicolumn{2}{|c|}{ Species } & \multirow{2}{*}{ Proportions } \\
\hline & abs. number & $\%$ & abs. number & $\%$ & abs. number & $\%$ & \\
\hline $\begin{array}{l}\text { Equisetophytal } \\
\text { Equisetopsida }\end{array}$ & 1 & 2,5 & 1 & 0,98 & 1 & 0,91 & 1:1:1 \\
\hline $\begin{array}{l}\text { Pinophytal } \\
\text { Pinopsida }\end{array}$ & 1 & 2,5 & 1 & 0,98 & 1 & 0,91 & 1:1:1 \\
\hline $\begin{array}{l}\text { Magnoliophytal } \\
\text { Magnoliopsida } \\
\text { Lilionsida }\end{array}$ & $\begin{array}{c}40 \\
4\end{array}$ & $\begin{array}{l}85,0 \\
10,0\end{array}$ & $\begin{array}{c}95 \\
5\end{array}$ & $\begin{array}{c}93,14 \\
4,90\end{array}$ & $\begin{array}{c}103 \\
5\end{array}$ & $\begin{array}{c}93,63 \\
4,55\end{array}$ & $\begin{array}{c}1: 2,8: 3 \\
1 \cdot 12 \cdot 12\end{array}$ \\
\hline Total: & 46 & 100 & 102 & 100 & 110 & 100 & $1: 2,5: 2,8$ \\
\hline
\end{tabular}

Tab.1. Taxonomical structure of Medicinal plants.

Magnoliophyta is the largest division by species richness. It includes 108 species $(98.18 \%$ of their total number). 103 species (93.63 \%) belong to Magnoliopsida. Divisions Equisetophyta and Pinophyta are represented by one species - Equisetum arvense L. та Juniperus communis L. respectively.

The predominant families are Asteraceae - 20 видів (18.18\% of their total number), Rosaceae - 10 видів (9.09\%), Lamiaceae - 9 видів (8.18\%). Two families contain six species each (5.45 \%) - Apiaceae i Polygonaceae (table 2). The families Solanaceae and Fabaceae include, respectively, 5 species (4.55\%) and 4 species (3.64 \%). Three species (2.73 \%) include Brassicaceae and Gentianaceae. Other families account for $40.0 \%$ of studied species, of which 30 families have only one species (Papaveraceae, Urticaceae, Caprifoliaceae, Plantaginaceae, Valerianaceae etc.). The species richness of families is 2.8.

\begin{tabular}{|c|c|c|c|c|c|}
\hline \multirow{2}{*}{$\begin{array}{c}\text { No in } \\
\text { order }\end{array}$} & \multirow{2}{*}{ Family } & \multicolumn{2}{|c|}{ Genus } & \multicolumn{2}{c|}{ Species } \\
\cline { 3 - 6 } & abs. number & $\%$ & abs. number & $\%$ \\
\hline 1. & Asteraceae & 19 & 18,63 & 20 & 18,18 \\
\hline 2. & Rosaceae & 8 & 7,84 & 10 & 9,09 \\
\hline 3. & Lamiaceae & 9 & 8,82 & 9 & 8,2 \\
\hline 4. & Apiaceae & 3 & 5,88 & 6 & 5,45 \\
\hline 5. & Polygonaceae & 5 & 4,94 & 6 & 5,45 \\
\hline 6. & Solanaceae & 50 & 5 & 4,55 \\
\hline
\end{tabular}

Tab. 2. Systematic analysis of main families. 
The vast majority of genera, namely 95 (93.14\% of the total number of genera), have a low level of floristic wealth and are single-species. Six genera (5.88\%) - Rumex L., Potentilla L., Ononis L., Gentiana L., Filipendula Mill., Artemisia L. include 2 species each, while the genus Polygonum L. includes 3 species.

The detailed results of the biomorphological analysis are represented on Figure 1. According to our data, polycarpic herbs form the largest group - 60 species (54.55\%) (Artemisia absintium L., A. vulgaris L., Gentiana lutea L., Salvia officinalis L., Lamium album L., Achillea submillefolium L., Hypericum perforatum L., Mentha piperita L., Hieracium villosum Jacq., Urtica dioica L., Aguilegia vulgaris L., Marrubium vulgare L., Potentilla anserina L., Valeriana exaltata Mihan, Equisetum arvense L., Pulmonaria officinalis L., Rheum rhaponticum L., Levisticum officinalis Koch etc.). The monocarpic herbs (annual plants) take the second place - 21 species (19.01\%) (Avena sativa L., Xanthium spinosum L., Chamomilla recutita L., Datura stramonium L., Apium graveolens L., Cucurbita pepo L., Saponaria officinalis L., Polygonum arviculare L. etc.). Trees are represented by nine species $(8,18 \%$ ) (Betula pendula Roth, Juglans regia L., Salix alba L., Populus nigra L. etc.). Less species are shrubs - eight species (7.27\%) (Arctostaphylos uva-ursi (L.) Spreng, Rosa canina L., Viburnum opulus L., Sambucus nigra L., Frangula alnus Mill., Prunus spinosa L., Ononis spinosa L., Viscum album L.). Seven species (6.36 \%) are biennial plants (Archahgelica officinalis Hoffm, Gentarium erythraea Rafn, Carum carvi L., Plantago major L., Allium sativum L., Hyoscyamus niger L., Verbascum phlomides L.). Such group as subshrubs are represented by four species (3.64 \%) (Vaccinium myrtillus L., Genista tinctoria L., Rhodococcum vitis-idaea Avror, Rubus caesius L.). And lianas take only one species $(0.91 \%)-$ Vitis vinifera L.

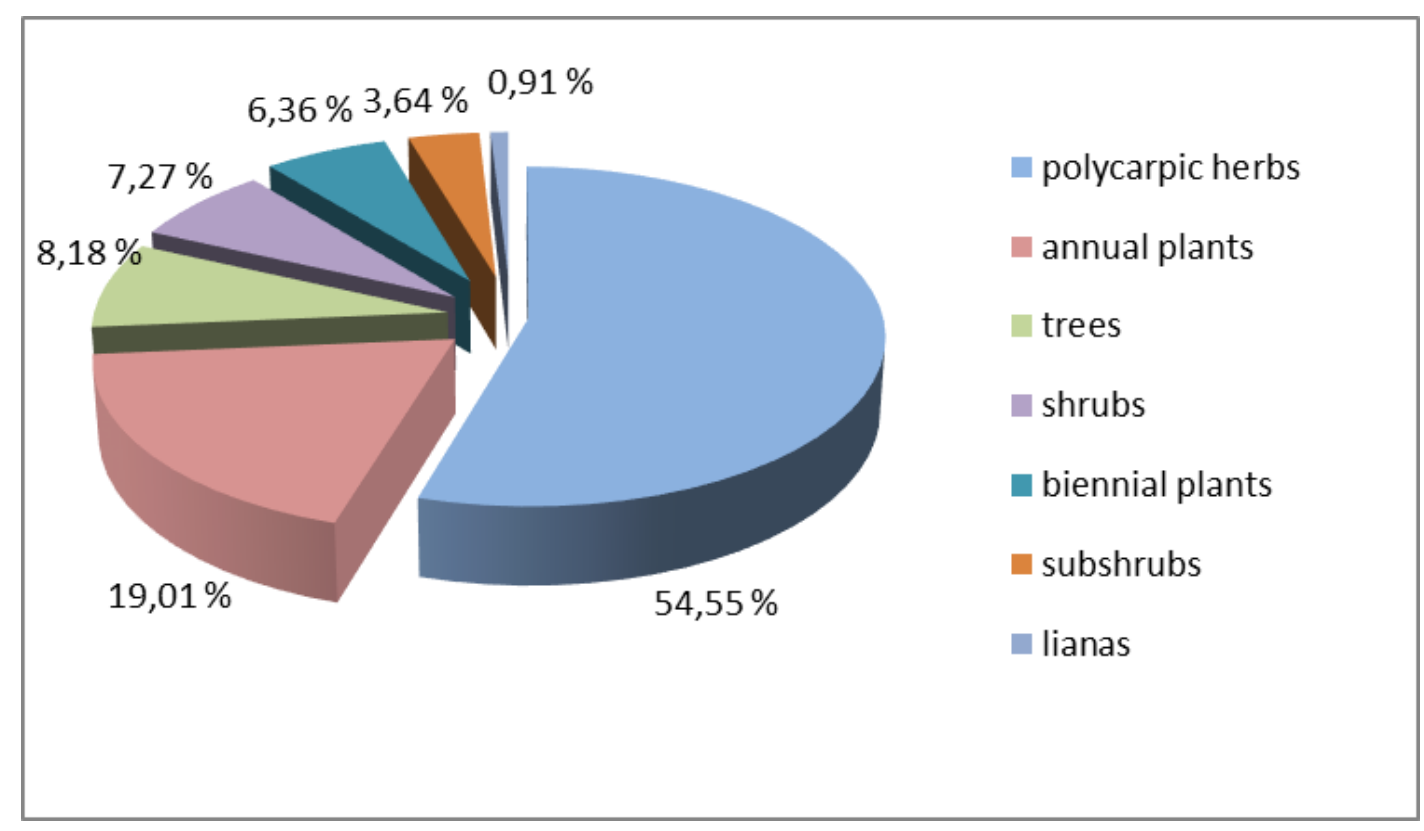

Fig. 1. The analysis of the plants' life-forms.

Diseases of the gastrointestinal tract are divided into four groups: stomach, intestines, liver and gallbladder and dyspeptic disorders [3].

60 species of studied plants belonging to 28 families are used for the treatment of gastric diseases. Among them, 3 families dominate (24.6\%): Asteraceae, Rosaceae, Lamiaceae. Biomorphological analysis showed a predominance of herbaceous polycarpics - 35 видів (58.33\%): Menyanthes trifoliata L., Mellissa officinalis L., Artemisia absintium L., Gentiana lutea L., Thymus serpyllum L., Hypericum perforatum L., Achillea submillefolium L., Filipendula vulgaris Moench, Symphytum officinale L., Solanum tuberosum L., Polemonicem caeruleum L. etc. The second place is occupied by annual plants - 10 species (16.67\%): Fumaria officinalis L., Cnicus benedictus L., Polygonum arviculare L., Bidens tripartite L., Calendula officinalis L., Chamomilla recutita L., Gnaphalium uliginosum L., Datura stramonium L., Euphrasia rostkoviana Hayne, Thlaspi arvense L. Biennial plants (Archahgelica officinalis Hoffm, Gentarium erythraea Rafn, Carum carvi L., 
Plantago major L., Allium sativum L.) and trees (Betula pendula Roth., Juniperus communis L., Juglans regia L., Berberis vulgaris L., Styphlonobium japonicum (L.) Schott.) are represented by five species each (8.33\%). Shrubs are a smaller group and have 4 species (6.67\%) - Arctostaphylos uva-ursi (L.) Spreng, Rosa canina L., Viburnum opulus L., Sambucus nigra L. The least numerous are lianas - 1 species (1.67\%): Vitis vivifera L.

The most common stomach diseases are atony, achillia, ulcers and gastritis (table 3). Gastric atony is a decrease or complete loss of gastric muscle tone, which can be congenital or acquired [5]. Such herbs are used for its treatment: Menyanthes trifoliata L., Carum carvi L., Linaria vulgaris Mill., Mellissa officinalis L., Arctostaphylos uva-ursi (L.) Spreng, Betula pendula Roth., Menyanthes trifoliata L., Archahgelica officinalis Hoffm, Gentarium erythraea Rafn etc.

Achillia - disorders of the secretory function of the gastric glands, a symptom of gastric disease (gastritis, cancer); under these conditions, you can use such plants: Polygonum arviculare L., Cichorium intybus L., Helichrysum avenarium (L.) Moench, Allium sativum L., Carum carvi L., Artemisia absintium L., Gentiana lutea L., Cnicus benedictus L., Armoracia rusticana Gaertn, Juniperus communis L., Acorus calamus L., Berberis vulgaris L., Hypericum perforatum L., Taraxacum officinale L., Mentha piperita L., Inula helenium L., Tanacetum vulgare L., Rosmarinus officinalis L., Ruta hortensis L., Rosa canina L. etc.

Gastritis - inflammation of the gastric mucosa, which causes a violation of its function [3]. For this disease, preference is given to the following species of plants: Fumaria officinalis L., Gentiana cruciata L., Acorus calamus L., Hypericum perforatum L., Plantago major L., Rosmarinus officinalis L., Polygonum arviculare L., Cichorium intybus L., Altheae officinalis L., Atropa bella-donna L., Viburnum opulus L., Chamomilla recutita L., Gnaphalium uliginosum L., Tussilago farfara L., Gleochoma hederaceae L., Thlaspi arvense L. etc.

Gastric ulcer - damage to the gastric mucosa, accompanied by the appearance of a defect in it, which does not heal for a long time [3]. Following species of plants are often used for its treatment: Betula pendula Roth., Acorus calamus L., Achillea submillefolium L., Altheae officinalis L., Symphytum officinale L., Viburnum opulus L., Solanum tuberosum L., Calendula officinalis L., Chamomilla recutita L., Polemonicem caeruleum L., Gnaphalium uliginosum L. etc.

The largest number of analyzed species - 75 (68.2\%), which belong to 36 families are used for dyspeptic disorders. The dominant families are: Asteraceae, Rosaceae, Apiaceae, Polygonaceae. Among the life forms, polycarpics predominate - 37 species (49.33\%): Mellissa officinalis L., Artemisia absintium L., Gentiana lutea L., Acorus calamus L., Lamium album L., Linaria vulgaris Mill., Achillea submillefolium L., Hypericum perforatum L., Taraxacum officinale L., Mentha piperita L., Inula helenium L., Tanacetum vulgare L., Artemisia vulgaris L., Ruta hortensis L., Altheae officinalis L., Equisetum arvense L., Angelina sylvestris L., Ononis arvensis L., Levisticum officinalis Koch. etc. Annual plants are represented by 13 species (17.33 \%): Fumaria officinalis L., Polygonum arviculare L., Chamomilla recutita L., Gnaphalium uliginosum L., Capsella bursa-pastoris (L.) Medik, Polygonum hydropiper L., Xanthium spinosum L., Avena sativa L. etc. Trees include eight species (10.67 \%) - Betula pendula Roth., Juniperus communis L., Berberis vulgaris L., Juglans regia L., Salix alba L., Quercus robur L., Aesculus hippocastanum L., Populus nigra L. Biennial plants and shrubs are represented by seven species each (9.33\%) - Archahgelica officinalis Hoffm, Gentarium erythraea Rafn, Carum carvi L., Plantago major L., Allium sativum L., Hyoscyamus niger L., Verbascum phlomides L. and Arctostaphylos uva-ursi (L.) Spreng, Rosa canina L., Viburnum opulus L., Sambucus nigra L., Frangula alnus Mill., Ononis spinosa L., Viscum album L. Three species belong to subshrubs (4.0\%) Genista tinctoria L., Rhodococcum vitis-idaea Avror, Rubus caesius L.

Dyspeptic disorders include: diarrhoea, bloating, nausea, vomiting, constipation. Diarrhoea is a disorder that is accompanied by frequent bowel movements [3]. To suppress the secretory and digestive activity of the intestines, as antidiarrheals, should be used: Archahgelica officinalis Hoffm, Gentarium erythraea Rafn, Arctostaphylos uva-ursi (L.) Spreng, Artemisia absintium L., Salvia officinalis L., Acorus calamus L., Berberis vulgaris L., Lamium album L., Juglans regia L., Tanacetum vulgare L., Polygonum arviculare L., Sambucus nigra L., Geum urbanum L., Ledum palustre L., Fragaria vesca L., Frangula alnus Mill., Rhodococcum vitis-idaea Avror, Rubus caesius L., Potentilla erecta (L.) Raeusch., Valeriana exaltata Mihan, Polygonum hydropiper L., Rheum rhaponticum L. etc. 
Bloated abdomen - a dyspeptic condition with excessive flatulence [12], for the treatment of which some herbs are recommended. They include: Carum carvi L., Linaria vulgaris Mill., Mellissa officinalis L., Gentiana lutea L., Gentiana cruciata L., Salvia officinalis L., Juniperus communis L., Inula helenium L., Ruta hortensis L., Allium sativum L., Chamomilla recutita L., Arnica montana L., Anethum graveolens L., Apium graveolens L., Avena sativa L., Levisticum officinalis Koch etc.

Vomiting - unauthorized secretion of gastric contents through the mouth [4]. The following plant species are effective: Betula pendula Roth., Menyanthes trifoliata L., Archahgelica officinalis Hoffm, Gentarium erythraea Rafn, Acorus calamus L., Lamium album L., Juglans regia L., Achillea submillefolium L., Hypericum perforatum L., Mentha piperita L., Hyoscyamus niger L., Valeriana exaltata Mihan, Equisetum arvense L., Angelina sylvestris L., Cucurbita pepo L.

Nausea is a pathological condition in which there are frequent calls for vomiting [5]. To eliminate this condition such plants are used: Lamium album L., Archahgelica officinalis Hoffm, Juglans regia L., Gentarium erythraea Rafn, Acorus calamus L., Achillea submillefolium L., Hypericum perforatum L., Mentha piperita L., Hyoscyamus niger L., Valeriana exaltata Mihan, Angelina sylvestris L. etc.

Constipation - a delay in the excretion of intestinal contents for one or more days. To overcome it you may use laxative plants containing fiber, pectin and hemicellulose [6] - Menyanthes trifoliata L., Taraxacum officinale L., Viburnum opulus L., Sambucus nigra L., Capsella bursa-pastoris (L.) Medik, Genista tinctoria L., Frangula alnus Mill., Rhodococcum vitis-idaea Avror, Ononis spinosa L., Ononis arvensis L., Verbascum phlomides L., Anethum graveolens L.

In liver disease, the formation and secretion of bile is disturbed, so you should use choleretic plants, the active substances of which are essential oils, phytosterols, flavonoids, resins, etc. [1]. The most common diseases of the liver and gallbladder are hepatitis $\mathrm{A}$, cholangitis and cholecystitis. For the treatment of these diseases 47 species of studied plants (42.73\%) from 26 families are used. The most numerous of them were the following families: Asteraceae, Rosaceae, Lamiaceae. The dominant group is herbaceous polycarpic plants - 32 species (68.08\%): Menyanthes trifoliata L., Linaria vulgaris Mill., Agrimonia eupatoria L., Artemisia absintium L., Gentiana lutea L., Gentiana cruciata L., Armoracia rusticana Gaertn, Acorus calamus L., Hypericum perforatum L., Mentha piperita L., Hieracium villosum Jacq., Inula helenium L., Tanacetum vulgare L., Rosmarinus officinalis L., Ruta hortensis L. etc. There are eight species of annual plants (17.02\%): Fumaria officinalis L., Cnicus benedictus L., Calendula officinalis L., Euphrasia rostkoviana Hayne, Centaurea cyanus L., Capsella bursa-pastoris (L.) Medik, Zea mays L., Saponaria officinalis L.; three species of trees (6.38 \%): Betula pendula Roth., Berberis vulgaris L., Salix alba L.; two species of shrubs (4.26 \%): Rosa canina L., Frangula alnus Mill.; one species (2.13\%) of biennial plants and subshrubs: Gentarium erythraea Rafn та Genista tinctoria L.

Cholangitis is a nonspecific inflammation of the bile ducts due to the penetration of infections from the gallbladder, intestines, blood and lymphatic vessels [3]. To treat this disease Tanacetum vulgare L., Cichorium intybus L., Solidago virgaurea L., Zea mays L., Urtica dioica L., Aguilegia vulgaris L. are used.

Hepatitis A, B, C - infectious diseases with liver damage caused by relevant viruses [4]. The following plants are used to treat hepatitis A: Agrimonia eupatoria L., Artemisia absintium L., Fumaria officinalis L., Gentiana lutea L., Armoracia rusticana Gaertn, Berberis vulgaris L., Hypericum perforatum L., Mentha piperita L., Inula helenium L., Tanacetum vulgare L., Rosmarinus officinalis L., Ruta hortensis L., Cichorium intybus L., Rosa canina L., Calendula officinalis L., Chelidonium major L., Geum urbanum L., Genista tinctoria L., Solidago virgaurea L., Asarum europaeum L., Zea mays L., Fragaria vesca L., Rumex acetosa L. etc.

Cholecystitis is an inflammation of the gallbladder wall [2]. This disease is treated with plants such as: Gentarium erythraea Rafn, Agrimonia eupatoria L., Fumaria officinalis L., Acorus calamus L., Berberis vulgaris L., Cichorium intybus L., Helichrysum avenarium (L.) Moench, Rosa canina L., Atropa bella-donna L., Capsella bursa-pastoris (L.) Medik, Zea mays L., Frangula alnus Mill., Hepatica nobilis Mill., Marrubium vulgare $\mathrm{L}$.

Intestinal disorders include enteritis and colitis, for the treatment of which 28 species $(25.45 \%)$ of studied plants from 17 families are used. The most numerous families are Asteraceae and Rosaceae. Among biomorphs, herbaceous polycarpic plants are represented by 15 species ( $53.57 \%)$ : Gentiana lutea 
L., Gentiana cruciata L., Salvia officinalis L., Hypericum perforatum L., Hieracium villosum Jacq., Tanacetum vulgare L., Altheae officinalis L., Geum urbanum L., Gleochoma hederaceae L., Ledum palustre L., Eriophorum latifolium Hoppe, Rumex confertus Willd., Arnica montana L., Potentilla anserina L., Potentilla erecta (L.) Raeusch. Trees include four species (14.29\%): Berberis vulgaris L., Juglans regia L., Salix alba L., Quercus robur L. Shrubs and subshrubs have three species (10.71\%) - Arctostaphylos uva-ursi (L.) Spreng, Viburnum opulus L., Prunus spinosa L. та Vaccinium myrtillus L., Rhodococcum vitis-idaea Avror, Rubus caesius L., respectively. Annual plants are represented by two species $(7.14 \%)$ - Chamomilla recutita L., Euphrasia rostkoviana Hayne. And biennials have only 1 species (3.57 \%) - Plantago major L.

Enteritis - inflammation of the mucous membrane of the small intestine [4]. The following species of medicinal plants are used: Plantago major L., Altheae officinalis L., Chamomilla recutita L., Ledum palustre L., Eriophorum latifolium Hoppe, Vaccinium myrtillus L., Rumex confertus Willd.

Colitis - inflammation of the mucous membrane of the colon; the use of such plants will be healing: Arctostaphylos uva-ursi (L.) Spreng, Gentiana lutea L., Gentiana cruciata L., Salvia officinalis L., Berberis vulgaris L., Juglans regia L., Hypericum perforatum L., Hieracium villosum Jacq., Tanacetum vulgare L., Plantago major L., Viburnum opulus L., Chamomilla recutita L., Gleochoma hederaceae L., Eriophorum latifolium Hoppe, Arnica montana L., Salix alba L., Rubus caesius L., Potentilla erecta (L.) Raeusch., Prunus spinosa L. etc.

\begin{tabular}{|c|c|c|c|c|c|c|c|c|c|c|c|c|c|c|}
\hline \multirow[t]{3}{*}{ Species } & \multicolumn{14}{|c|}{ Diseases of the gastrointestinal tract } \\
\hline & \multicolumn{4}{|c|}{ stomach } & \multicolumn{2}{|c|}{ intestine } & \multicolumn{4}{|c|}{ liver and gallbladder } & \multicolumn{4}{|c|}{ dyspeptic disorders } \\
\hline & 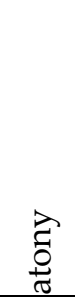 & 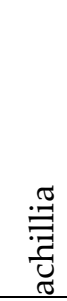 & $\frac{\ddot{d}}{3}$ & 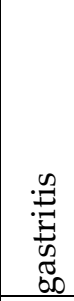 & 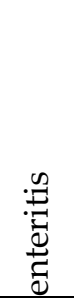 & $: \stackrel{0}{: 0}$ & 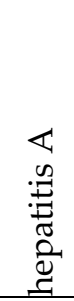 & 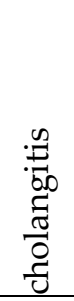 & 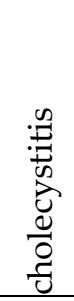 & $\begin{array}{l}\mathbb{J} \\
\mathscr{W} \\
\mathbb{\Xi} \\
\Xi\end{array}$ & 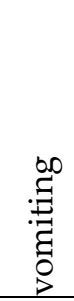 & 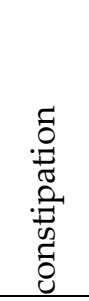 & 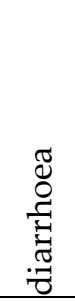 & 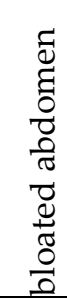 \\
\hline Betula pendula Roth. & + & & + & & & & + & & & + & + & & & \\
\hline Menyanthes trifoliata L. & + & + & & + & & & + & & & + & + & + & & \\
\hline Archahgelica officinalis Hoffm & + & & & & & & & & & + & + & & + & + \\
\hline Gentarium erythraea Rafn & + & + & & + & & & & & + & + & + & & + & + \\
\hline Carum carvi L. & + & + & & + & & & & & & & & & & + \\
\hline Linaria vulgaris Mill. & + & & & & & & + & & & & & & & + \\
\hline Mellissa officinalis L. & + & & & & & & & & & & & & & + \\
\hline $\begin{array}{l}\text { Arctostaphylos uva-ursi (L.) } \\
\text { Spreng }\end{array}$ & + & & & & & + & & & & & & & + & \\
\hline Agrimonia eupatoria L. & + & & & & & & + & & + & & & & & \\
\hline Artemisia absintium L. & + & + & & + & & & + & & & & & & + & + \\
\hline Fumaria officinalis L. & + & + & + & + & & & + & & + & & & & & + \\
\hline Gentiana lutea L. & + & + & & + & & + & + & & & & & & & + \\
\hline Gentiana cruciata L. & + & + & & + & & + & + & & & & & & & + \\
\hline Cnicus benedictus L. & + & + & & & & & + & & & & & & & \\
\hline Armoracia rusticana Gaertn & + & + & & & & & + & & & & & & & \\
\hline Thymus serpyllum L. & + & & & & & & & & & & & & & \\
\hline Salvia officinalis L. & + & & + & & & + & & & & & & & + & + \\
\hline Juniperus communis L. & + & + & & & & & & & & & & & & + \\
\hline Acorus calamus $\mathrm{L}$. & & + & + & + & & & & & + & + & + & & + & + \\
\hline Berberis vulgaris L. & & + & & & & + & + & & + & & & & + & \\
\hline Vitis vinifera $\mathrm{L}$. & & + & & & & & & & & & & & & \\
\hline
\end{tabular}




\begin{tabular}{|c|c|c|c|c|c|c|c|c|c|c|c|c|c|}
\hline Lamium album $\mathrm{L}$. & + & & & & & & & & + & + & & + & \\
\hline Juglans regia L. & + & & & & + & & & & + & + & & + & \\
\hline Achillea submillefolium $\mathrm{L}$. & + & + & + & & & & & & + & + & & + & \\
\hline Hypericum perforatum L. & + & & + & & + & + & & & + & + & & + & \\
\hline Taraxacum officinale $\mathrm{L}$. & + & & + & & & & & & & & + & & \\
\hline Mentha piperita L. & + & & + & & & + & & & + & + & & & + \\
\hline Hieracium villosum Jacq. & + & + & & & + & + & & & & & & & \\
\hline Inula helenium L. & + & & & & & + & & & & & & & + \\
\hline Tanacetum vulgare $\mathrm{L}$. & + & & & & + & + & + & & & & & + & \\
\hline Plantago major L. & + & & + & + & + & & & & & & & + & \\
\hline Artemisia vulgaris L. & + & & & & & & & & & & & + & \\
\hline Rosmarinus officinalis L. & + & & + & & & + & & & & & & & \\
\hline Ruta hortensis L. & + & & & & & + & & & & & & & + \\
\hline Polygonum arviculare L. & + & & + & & & & & & & & & + & \\
\hline Cichorium intybus L. & + & & + & & & + & + & + & & & & & \\
\hline $\begin{array}{l}\text { Helichrysum avenarium (L.) } \\
\text { Moench }\end{array}$ & + & & & & & + & & + & & & & & \\
\hline Allium sativum $\mathrm{L}$. & + & & & & & & & & & & & & + \\
\hline Bidens tripartite $\mathrm{L}$. & + & & & & & & & & & & & & \\
\hline Rosa canina $\mathrm{L}$. & + & + & & & & + & & + & & & & + & \\
\hline Altheae officinalis L. & & + & + & + & + & & & & & & & + & \\
\hline Atropa bella-donna L. & & + & + & & & & & + & & & & & \\
\hline Filipendula vulgaris Moench & & + & & & & & & & & & & + & \\
\hline Symphytum officinale L. & & + & + & & & & & & & & & + & \\
\hline Viburnum opulus L. & & + & + & & + & & & & & & + & + & \\
\hline Solanum tuberosum L. & & + & + & & & & & & & & & & \\
\hline Calendula officinalis L. & & + & & & & + & & & & & & & \\
\hline Chamomilla recutita $\mathrm{L}$. & & + & + & + & + & & & & & & & + & + \\
\hline Polemonicem caeruleum L. & & + & & & & & & & & & & & \\
\hline $\begin{array}{l}\text { Styphlonobium japonicum (L.) } \\
\text { Schott }\end{array}$ & & + & & & & & & & & & & & \\
\hline Gnaphalium uliginosum L. & & + & + & & & & & & & & & & + \\
\hline $\begin{array}{l}\text { Filipendula denudata (J. et C. } \\
\text { Presl.) Fritsch }\end{array}$ & & & + & & & & & & & & & + & \\
\hline Chelidonium major $\mathrm{L}$. & & & + & & & + & & & & & & & \\
\hline Sambucus nigra L. & & & + & & & & & & & & + & + & \\
\hline Geum urbanum L. & & & + & & + & + & & & & & & + & \\
\hline Datura stramonium L. & & & + & & & & & & & & & & \\
\hline Euphrasia rostkoviana Hayne & & & + & & + & + & & & & & & & + \\
\hline Tussilago farfara $\mathrm{L}$. & & & + & & & & & & & & & & \\
\hline Gleochoma hederaceae L. & & & + & & + & & & & & & & & \\
\hline Thlaspi arvense L. & & & + & & & & & & & & & & \\
\hline Ledum palustre $\mathrm{L}$. & & & & + & + & & & & & & & + & \\
\hline Eriophorum latifolium Hoppe & & & & + & + & & & & & & & & \\
\hline Vaccinium myrtillus L. & & & & + & + & & & & & & & & \\
\hline Rumex confertus Willd. & & & & + & + & & & & & & & + & \\
\hline Arnica montana L. & & & & & + & + & & & & & & & + \\
\hline Salix alba $\mathrm{L}$. & & & & & + & + & & & & & & + & \\
\hline Verbena officinalis L. & & & & & & + & & & & & & & \\
\hline Centaurea cyanus L. & & & & & & + & & & & & & & \\
\hline $\begin{array}{l}\text { Capsella bursa-pastoris (L.) } \\
\text { Medik }\end{array}$ & & & & & & + & & + & & & + & & \\
\hline
\end{tabular}


The Structural Analysis of Medicinal Plants and Their Use to Treat... 73

\begin{tabular}{|c|c|c|c|c|c|c|c|c|c|c|c|c|c|c|}
\hline Genista tinctoria L. & & & & & & & + & & & & & + & & \\
\hline Solidago virgaurea L. & & & & & & & + & + & & & & & & \\
\hline Asarum europaeum L. & & & & & & & + & & & & & & & \\
\hline Antennaria dioica (L.) Gaertn & & & & & & & + & & & + & + & & & \\
\hline Zea mays L. & & & & & & & + & + & + & & & & & \\
\hline Origanum vulgare L. & & & & & & & + & & & & & & & \\
\hline Saponaria officinalis L. & & & & & & & + & & & & & & & \\
\hline Fragaria vesca L. & & & & & & & + & & & & & & + & \\
\hline Rumex acetosa $\mathrm{L}$. & & & & & & & + & & & & & & & \\
\hline Urtica dioica L. & & & & & & & & + & & & & & & \\
\hline Aguilegia vulgaris L. & & & & & & & & + & & & & & & \\
\hline Frangula alnus Mill. & & & & & & & & & + & & & + & + & \\
\hline Hepatica nobilis Mill. & & & & & & & & & + & & & & & \\
\hline Marrubium vulgare L. & & & & & & & & & + & & & & + & \\
\hline Quercus robur L. & & & & & & + & & & & & & & + & \\
\hline $\begin{array}{ll}\text { Rhodococcum vitis-idaea } \\
\text { Avror }\end{array}$ & & & & & & + & & & & & & + & + & \\
\hline Rubus caesius L. & & & & & & + & & & & & & & + & \\
\hline Potentilla anserina L. & & & & & & + & & & & & & & & \\
\hline Potentilla erecta (L.) Raeusch. & & & & & & + & & & & & & & + & \\
\hline Prunus spinosa L. & & & & & & + & & & & & & & & \\
\hline Hyoscyamus niger L. & & & & & & & & & & + & + & & & \\
\hline Valeriana exaltata Mihan & & & & & & & & & & + & + & & + & + \\
\hline Equisetum arvense L. & & & & & & & & & & & + & & + & \\
\hline Angelina sylvestris L. & & & & & & & & & & + & + & & + & + \\
\hline Cucurbita pepo L. & & & & & & & & & & + & + & & & \\
\hline $\begin{array}{ll}\text { Linum crepitans (Boenn.) } \\
\text { Dumort. }\end{array}$ & & & & & & & & & & & & + & & \\
\hline Ononis spinosa L. & & & & & & & & & & & & + & & \\
\hline Ononis arvensis $\mathrm{L}$. & & & & & & & & & & & & + & & \\
\hline Verbascum phlomides L. & & & & & & & & & & & & + & & \\
\hline Anethum graveolens L. & & & & & & & & & & & & + & & + \\
\hline Aesculus hippocastanum L. & & & & & & & & & & & & & + & \\
\hline Polygonum bistorta L. & & & & & & & & & & & & & + & \\
\hline Polygonum hydropiper L. & & & & & & & & & & & & & + & \\
\hline Pulmonaria officinalis L. & & & & & & & & & & & & & + & \\
\hline Xanthium spinosum L. & & & & & & & & & & & & & + & \\
\hline Viscum album L. & & & & & & & & & & & & & + & \\
\hline Apium graveolens L. & & & & & & & & & & & & & & + \\
\hline Populus nigra L. & & & & & & & & & & & & & + & \\
\hline Rheum rhaponticum L. & & & & & & & & & & & & & + & \\
\hline Avena sativa L. & & & & & & & & & & & & & & + \\
\hline Levisticum officinalis Koch & & & & & & & & & & & & & & + \\
\hline Total: & 18 & 32 & 18 & 32 & 7 & 28 & 39 & 6 & 14 & 15 & 16 & 13 & 45 & 26 \\
\hline
\end{tabular}

Tab. 3. The use of medicinal plants for diseases of the gastrointestinal tract. 


\section{Conclusions}

As a result of the analysis of literature sources for the treatment of diseases of the digestive system 110 species of plants belonging to 102 genera, 46 families, 33 orders, 4 classes (Equisetopsida, Pinopsida, Magnoliopsida Liliopsida) and 3 divisions (Equisetophyta, Pinophyta та Magnoliophyta) were revealed.

The predominant families are Asteraceae - 20 species (18.18\% of the total number of species), Rosaceae - 10 species (9.09\%), Lamiaceae - 9 species (8.18\%), Apiaceae - 6 species (5.45\%) i Polygonaceae 6 species $(5.45 \%)$. The species richness of families is 2.8 .

Among biomorphs medicinal plants were distributed: herbaceous polycarpic - 60 species $(54.55 \%)$, annual plants - 21 species (19.01\%), trees - 9 species $(8.18 \%)$, shrubs - 8 species $(7.27) \%)$, biennial plants - 7 species $(6.36 \%)$, subshrubs - 4 species $(3.64 \%)$, lianas - 1 species $(0.91 \%)$.

60 species of medicinal plants are effective in gastric disease, 28 species in the intestine, 47 species in the liver and gallbladder, and 75 species in dyspeptic disorders.

In various diseases of the gastrointestinal tract are most often used such plant species as: Gentarium erythraea Rafn, Acorus calamus L., Menyanthes trifoliata L., Fumaria officinalis L., Hypericum perforatum L., Artemisia absintium L., Gentiana lutea L., Gentiana cruciata L., Achillea submillefolium L., Mentha piperita L., Chamomilla recutita L., Betula pendula Roth., Archahgelica officinalis Hoffm, Salvia officinalis L., Berberis vulgaris L., Juglans regia L., Tanacetum vulgare L., Plantago major L., Cichorium intybus L., Rosa canina L., Altheae officinalis L., Viburnum opulus L.

\section{REFERENCES}

[1] Annamukhamedova O.O., Annamukhamedov A.O. Medicinal plants: a textbook. Zhytomyr, ZhDU of I. Franko, 2014. (in Ukrainian)

[2] Boltarevych Z.Ye. Ukrainians` folk medicine. Kyiv, Naukova dumka, 1990. (in Ukrainian)

[3] Glushko L.V. et al. Internal diseases: a textbook. Ivano-Frankivsk: Ivano-Frankivska derzhavna medychna academia, 2004. (in Ukrainian)

[4] Grodzinskyi A.M. Medicinal plants: encyclopedic reference book. Kyiv, Golovna redaktsia URE, 1990. (in Ukrainian)

[5] Zynchenko T.V., Stakhiv I.V., Miakushko T.Ya. Medicinal plants in gastroenterology. Kyiv, Naukova dumka, 1990. (in Russian)

[6] Karhut V.V. Alive pharmacy. Kyiv, Zdorovia, 1992. (in Ukrainian)

[7] Minarchenko V.M., Tymchenko I.A. Atlas of Ukrainian medicinal plants. Kyiv, Fitisociotsentr, 2002. (in Ukrainian)

[8] Dobrochaeva D.N. et al. Determinant of higher plants of Ukraine. Kyiv, Fitisociotsentr, 1999. (in Russian)

[9] Serebriakov I.G. Ecological morphology of plants. Life forms of angiosperms. Moscow, Vusshaia shkola, 1962. (in Russian)

[10] Garna S.V. et al. Modern phytotherapy: a textbook. Kharkiv, Drukarnia Madryd, 2016. (in Ukrainian)

[11] Tahtadzhian A.L. Magnoliophyte system. Leningrad, 1987. (in Russian)

[12] Samura B.A. et al. Phytotherapy in the clinic of internal medicine. Kharkiv, Zolotye stranitsy, 2003. (in Russian)

Address: Liubov Makhovska, Victoria Gniezdilova, Oksana Nespliak, Vira Buniak, Vasyl Stefanyk Precarpathian National University, 57 Shevchenko St., Ivano- Frankivsk 76018, Ukraine.

E-mail: liubov.makhovska@pnu.edu.ua; viktoria.gniezdilova@pnu.edu.ua; oksana.nespliak@pnu.edu.ua; vira.buniak@pnu.edu.ua.

Received: 10.11.2020; revised: 25.12.2020. 
Маховська Аюбов, Гнєзділова Вікторія, Неспляк Оксана, Буняк Віра. Структурний аналіз дікарських рослин та застосування їх при захворюваннях органів шлунково-кишкового тракту. Журнал Прикарпатського університету імені Василя Стефаника, 7 (4) (2020), 66-75.

Лікарські рослини з глибокої давнини використовуються при дікуванні різних захворювань, і при хворобах органів травлення, зокрема. Вони містять майже всі біологічно активні речовини, що надає їм перевагу над синтетичними дікарськими препаратами, та мождивість застосування впродовж тривалого часу. На основі опрацювання літературних джерел проведено систематичний та біоморфологічний аналізи лікарських рослин, які застосовуються при захворюваннях органів шлунково-кишкового тракту, серед яких виділено чотири групи: шлунка (атонія, ахілія, виразка, гастрит), кишечника (ентерит, коліт), печінки та жовчного міхура (гепатит А, холангіт, холецистит, нудота) i диспептичні розлади (блювота, закрепи, діарея, вздуття). Кожна з цих груп характеризується певним видовим списком дікарських рослин із зазначенням життєвих форм. Виявлено 110 видів лікарських рослин, які відносяться до 102-х родів, 46-и родин, 33-х порядків, 4-х класів та 3-х відділів. Серед лікарських рослин найбільш чисельнішими є трав'яні полікарпики - 60 видів (54,55 \%), тоді як однорічники налічують 21 вид (19,01 \%), дерева - 9 видів $(8,18 \%)$, кущі - 8 видів (7,27 \%), дворічники- 7 видів (6,36 \%), напівкущі - 4 види (3,64 \%) та ліани - 1 вид $(0,91 \%)$. Таксономічну та біоморфологічну структури досліджуваних рослин подано загадом та детально проаналізовано в кожній із виділених груп хвороб. Наведено приклади рослин, які застосовують при лікуванні різних захворювань органів шиунково-кишкового тракту. Встановлено, що при захворюванні кишечника використовують 28 видів, печінки та жовчного міхура - 47 видів, шиунка - 60 видів та диспептичних розладах - 75 видів лікарських рослин. Для лікування різних захворюваннях шлунковокишкового тракту найчастіше використовуються такі види рослин, як: Gentarium erythraea Rafn, Acorus calamus L., Menyanthes trifoliata L., Fumaria officinalis L., Hypericum perforatum L., Artemisia absintium L., Gentiana lutea L., Gentiana cruciata L., Achillea submillefolium L., Mentha piperita L., Chamomilla recutita L., Betula pendula Roth., Archahgelica officinalis Hoffm, Salvia officinalis L., Berberis vulgaris L., Juglans regia L., Tanacetum vulgare L., Plantago major L., Cichorium intybus L., Rosa canina L., Altheae officinalis L., Viburnum opulus L.

Кдючові слова: лікарські рослини, систематична структура, біоморфологічна структура, шиунково-кишковий тракт. 